C2008 IEEE. Personal use of this material is permitted. However, permission to reprint/republish this material for advertising or promotional purposes or for creating new collective works for resale or redistribution to servers or lists, or to reuse any copyrighted component of this work in other works must be obtained from the IEEE. 


\title{
Space-Time Power Scheduling of MIMO Links-Fairness and QoS Considerations
}

\author{
Yue Rong, Member, IEEE, and Yingbo Hua, Fellow, IEEE
}

\begin{abstract}
Power control, beamforming and link scheduling are all important operations to improve the power-and-spectral efficiency of networks of multiple-input multiple-output (MIMO) wireless links. We call a joint optimization of the above operations the space-time power scheduling (STPS) scheme. The STPS scheme is formulated as joint optimization of the transmitter covariance matrices of all active MIMO links over all dimensions of space and time, which includes the dimension of frequency as a dual form of time. In this paper, we address the proportional fair (PF) and quality-of-service (QoS) issues of the STPS scheme, which are important for networks with asymmetric topology and/or asymmetric traffic demands. Both slow fading channels and fast fading channels are considered. We demonstrate that the PF-STPS scheme provides a very attractive tradeoff between sum capacity and rate distribution for asymmetric links. We also demonstrate that the QoS-STPS scheme has a much higher power-and-spectral efficiency than the previously existing QoS based scheme that do not exploit the temporal freedom. Efficient optimization algorithms for both PF-STPS and QoS-STPS are provided. The STPS scheme is a centralized cooperative scheme which requires a scheduler. For ad hoc networks, this scheduler can be elected adaptively among eligible nodes in the network.
\end{abstract}

Index Terms-MIMO systems, proportional fairness, quality-ofservice, space-time power scheduler.

\section{INTRODUCTION}

$\mathbf{T}$ HIS paper considers a network of distributed multiple-input multiple-output (MIMO) wireless links. The topology of the network can be arbitrary, an example of which is illustrated in Fig. 1. This network may represent a spectrally isolated portion of a much larger network. In fact, if the original network is too large, for ease of management, the larger network can be partitioned into multiple smaller networks, each of which is assigned with an orthogonal frequency band. The focus of this paper is to optimize the power-and-spectral efficiency of a given network in a given frequency band. Many conventional nondistributed MIMO links such as multiple access MIMO links (i.e., multiple users with multiple antennas transmitting

Manuscript received July 13, 2007; revised February 10, 2008. This work was supported in part by the U.S. National Science Foundation under Grant ECS-0401310, the U.S. Army Research Laboratory under the Collaborative Technology Alliance Program, and the U.S. Army Research Office under the MURI Grant W911NF-04-1-0224. The U.S. Government is authorized to reproduce and distribute reprints for Government purposes notwithstanding any copyright notation thereon. The associate editor coordinating the review of this manuscript and approving it for publication was Dr. Homayoun Yousefi'zadeh.

Y. Rong was with the Department of Electrical Engineering, University of California, Riverside, CA 92521. He is now with the Department of Electrical and Computer Engineering, Curtin University of Technology, Bentley, WA 6102, Australia (e-mail: y.rong@ curtin.edu.au).

Y. Hua is with the Department of Electrical Engineering, University of California, Riverside, CA 92521 (e-mail: yhua@ee.ucr.edu).

Digital Object Identifier 10.1109/JSTSP.2008.920667

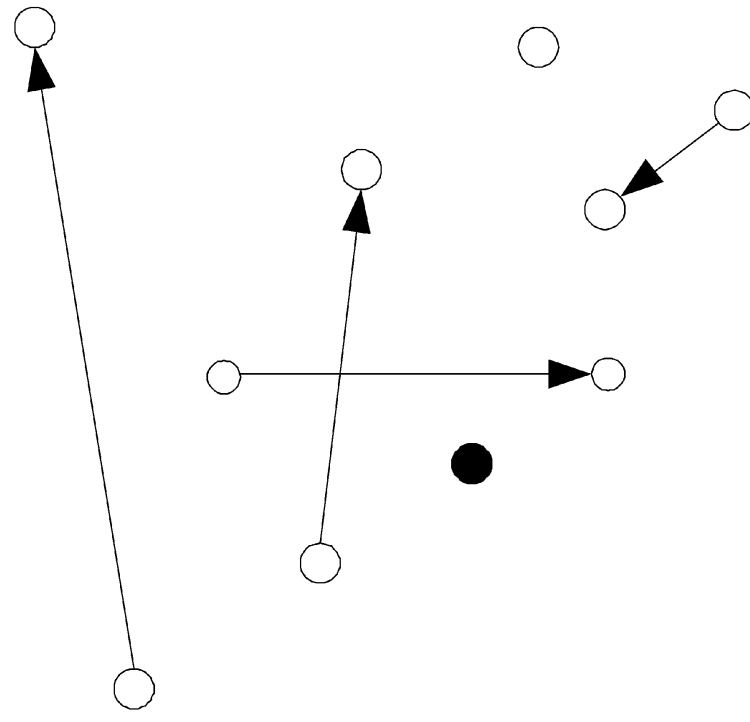

Fig. 1. Ad hoc network of distributed MIMO links where each node possibly has multiple antennas. The arrows indicate the desired active links in a time/ frequency band of interest. The black node is an elected scheduler. The other nodes are idle.

to a base station with multiple antenna) and broadcast MIMO links (i.e., a base station with multiple antennas transmitting to multiple users with multiple antennas) are extreme cases of the distributed MIMO links.

The power-and-spectral efficiency of the network of distributed MIMO links depends on the operations at all links in the network. To achieve a high power-and-spectral efficiency, a scheduler for the network is desirable whenever possible. For a cellular network, the scheduler should naturally reside at the base station. For an ad hoc network, the scheduler can reside at any node in the network. See Fig. 1. In a dynamic environment where nodes come and go, the scheduler can be adaptively elected among eligible nodes in the network. The election can be done based on the capacity of a node, the status of a node, and the location of a node, etc. The research of the scheduler election issues is important but beyond the scope of this paper. We assume that a scheduler is available for the network within the time scale of interest.

At the most ideal extreme, one would like to develop cooperative coding schemes for distributed MIMO links in order to maximize the network throughput. But this approach is currently considered to be highly infeasible even in theory. For a network of only two single-input single-output (SISO) links, cooperative coding is still considered a challenging research topic to date [1]. Because of this limitation, we will assume that only single-user decoder is used at each MIMO link. 
The next most fundamental problem for maximizing the network throughput (which is treated as an equivalent term for power-and-spectral efficiency) is to design the best transmitter covariance matrix of each MIMO link. Here and throughout the paper, we assume that the single-user receivers of all links are optimally designed. In [2]-[4], the authors investigated the optimal design of single-beam beamforming of each MIMO link (or equivalently joint design of transmitter and receiver beamforming). The single-beam beamforming approach effectively treats the transmitter covariance matrix of each MIMO link as a rank-one matrix. In [5]-[8], the network throughput under optimal transmitter covariance matrix without any rank constraint for each MIMO link is investigated. The best network throughput achievable by this approach is generally higher than that based on the single-beam beamforming approach. All of the above mentioned works can be seen as a generalization of power control from distributed SISO links to distributed MIMO links. However, they are all limited to a setting where each MIMO link is allocated with a time (or frequency) invariant transmitter covariance matrix. The above approaches do not treat link scheduling as an integral part of the design of transmitter covariance matrices.

In the recent works [9], [10], the authors proposed a joint optimization of power control, multiple-beam beamforming and link scheduling where effectively the transmitter covariance matrix of each link is treated as a time (or frequency) varying function within the given frequency band. This is what we call space-time power scheduling (STPS). Under a symmetric network condition, the STPS scheme has been developed for maximizing a sum capacity of distributed MIMO links for slow fading channels in [9] and for fast fading channels in [10].

In this paper, we further develop the STPS scheme by addressing the issues of fairness and quality-of-service (QoS). These issues are particularly important for networks with asymmetric topology and/or asymmetric traffic demands.

To address the fairness issue, we will incorporate a proportionally fair (PF) utility function into the STPS scheme. The PF notion was proposed in [11] and later also applied in [12]-[14]. The rates of all links are said to be proportionally fair if any increase of the rate of one link by $x \%$ would result in a decrease of the mean rate of other links by more than $x \%$. Remarkably, the PF notion is automatically embedded in such a utility function that is the sum of the logarithms of link rates. In this paper, we demonstrate that by incorporating the PF notion into the STPS scheme, the resulting PF-STPS scheme typically yields a near maximum sum capacity for asymmetric networks while a relatively even distribution of link rates can be maintained. Furthermore, by applying ([15], Theorem IV.1) for the fast fading case, we will develop a simpler form of the ergodic capacity, which in turn leads to a much simplified optimization algorithm than that in [10].

On the other hand, QoS corresponds to a set of predetermined service performance [16]. Mathematically, QoS can be formulated into different forms of constraints such as the signal-tointerference-plus-noise ratio (SINR) constraint [2], [3], the transmission error rate constraint [17], and the end-to-end rate constraint [18]. In this paper, we will consider a set of link-wise rate constraints as the QoS measure. Such a rate-constrained
STPS scheme will be referred to as the QoS-STPS scheme. Under the QoS-STPS scheme, any set of finite data rates of all links is feasible. This property does not hold for the space-only power scheduling scheme such as [7], where the feasible region of data rates is bounded even if the transmission power of each link is unbounded. Since any finite data rates are achievable with finite power under the STPS scheme, we can apply the penalty method ([19, Sec. 3.1]) to develop a very efficient optimization algorithm. As tested in simulations, this algorithm consistently yields the results that satisfy the desired rates.

It is important to note that under the STPS scheme, the mathematical problem is nonconvex. All known attempts to simplify the problem into a convex problem result in loss of power-andspectral efficiency. The optimization algorithm that we have developed for the STPS scheme is a projected gradient search. The transmitter covariance matrices from any other scheme can be used as an initial condition in the STPS based search. Although not guaranteed to be "globally optimal", the results from the STPS based search are consistently better than those under other schemes. This has been shown in [9] and [10] and will be further demonstrated in this paper. For convenience, we will not always distinguish the STPS scheme from the STPS based search algorithms.

The rest of this paper is organized as follows. In Section II, the preliminaries of the STPS scheme are introduced. In Section III, we present the PF-STPS scheme. In Section IV, we develop the QoS-STPS scheme. The performance of the PF-STPS scheme and the QoS-STPS scheme is illustrated in Section V.

\section{Preliminaries of the STPS SCHEME}

We consider a network of $L$ distributed MIMO links operating in a given frequency band. Each link consists of a transmitting node and a receiving node, and no link shares a node with another link. In practice, such a network represents a snapshot of an ad hoc network where the pairing of transceivers has been determined from an upper layer operation.

Both slow fading channels and fast fading channels will be considered. For slow fading channels, the channel coefficients remain constant within the time window $W_{D}$ of a complete packet as governed by a delay requirement in practice. For fast fading channels, the channel coefficients change randomly within $W_{D}$. But for both cases, we assume that the channel coefficients remain constant over $T \geq L$ contiguous time slots. The length of each time slot can vary significantly, which depends on many factors including the available bandwidth. The "time slots" referred to in this paper can be replaced by "frequency bins" or a combination of "time and frequency". This will not change the power-and-spectral efficiency of the STPS scheme.

The transmitter covariance matrix of each link is treated as a function of the time slot index $t$, which is denoted by $\mathbf{P}_{i}(t)$ with $i=1, \ldots, L$ and $t=1, \ldots, T$. For slow fading channels, $W_{D}$ can be set to equal $T$ time slots. For fast fading channels, $W_{D}$ can be set to equal a large multiple of $T$ time slots. The development of a search algorithm to compute $\mathbf{P}_{i}(t)$, for $i=$ $1, \ldots, L$ and $t=1, \ldots, T$, is a central part of the STPS scheme.

We will refer to the receiver of the $i$ th desired (as opposed to interfering) link as the $i$ th receiver and the transmitter of the $i$ th 
desired link as the $i$ th transmitter. The signal $\mathbf{y}_{i}(t)$ received by the $i$ th receiver during the $t$ th time slot is modeled as

$$
\begin{array}{r}
\mathbf{y}_{i}(t)=\sqrt{\beta_{i, i}} \mathbf{H}_{i, i} \mathbf{x}_{i}(t) \\
+\sum_{j=1, j \neq i}^{L} \sqrt{\beta_{i, j}} \mathbf{H}_{i, j} \mathbf{x}_{j}(t)+\mathbf{v}_{i}(t)
\end{array}
$$

where $\mathbf{H}_{i, j}$ is the $N \times N$ channel matrix between the $j$ th transmitter and the $i$ th receiver, $\beta_{i, j}$ denotes the channel gain (including the propagation path-loss and shadowing effect) between the $j$ th transmitter and the $i$ th receiver, $\mathbf{x}_{i}(t)$ is the signal vector transmitted from the $i$ th transmitting node within the $t$ th time slot, and $\mathbf{v}_{i}(t)$ is the noise. The covariance matrix of $\mathbf{x}_{i}(t)$ is $\mathbf{P}_{i}(t)$, i.e., $\mathbf{P}_{i}(t) \triangleq \mathrm{E}\left\{\mathbf{x}_{i}(t) \mathbf{x}_{i}^{H}(t)\right\}$ where $\mathrm{E}\{\cdot\}$ is the expectation. We assume that $\mathbf{v}_{i}(t)$, for $i=1, \ldots, L$ and $t=1, \ldots, T$, are independent and identically distributed (i.i.d.) Gaussian noise (AWGN) with zero mean and covariance matrix $\mathbf{C}_{\mathbf{v}_{i}}=\mathbf{I}_{N}$. Here $\mathbf{I}_{N}$ denotes the $N \times N$ identity matrix. We have assumed for convenience that each node has $N$ antennas.

The capacity of the $i$ th link over $T$ time slots is given by

$$
I_{i}(\mathcal{P})=\frac{1}{T} \sum_{t=1}^{T} \log _{2}\left|\mathbf{I}_{N}+\beta_{i, i} \mathbf{H}_{i, i} \mathbf{P}_{i}(t) \mathbf{H}_{i, i}^{H} \mathbf{R}_{i}^{-1}(t)\right|
$$

where $\mathcal{P} \triangleq\left\{\mathbf{P}_{i}(t), i=1, \ldots, L, t=1, \ldots, T\right\},|\cdot|$ denotes the determinant of a matrix, and $\mathbf{R}_{i}(t)$ is the interference-plusnoise covariance matrix at the $i$ th receiving node within the $t$ th time slot, i.e.,

$$
\mathbf{R}_{i}(t)=\sum_{j=1, j \neq i}^{L} \beta_{i, j} \mathbf{H}_{i, j} \mathbf{P}_{j}(t) \mathbf{H}_{i, j}^{H}+\mathbf{I}_{N} .
$$

In [9], the following STPS scheme is proposed:

$$
\begin{array}{ll}
\max _{\mathcal{P}} & \sum_{i=1}^{L} I_{i}(\mathcal{P}) \\
\text { s.t. } & \frac{1}{T} \sum_{t=1}^{T} \operatorname{tr}\left\{\mathbf{P}_{i}(t)\right\}=P_{i}, \quad i=1, \ldots, L \\
& \mathbf{P}_{i}(t) \geq 0, \quad i=1, \ldots, L, \quad t=1, \ldots, T
\end{array}
$$

where $\operatorname{tr}\{\cdot\}$ denotes the trace of a matrix. This scheduling problem is useful for slow fading channels where all channel matrices are required by the scheduler. The receiver of each link needs to know the channel matrix of that link.

In [10], the STPS scheme is extended to fast fading channels as follows:

$$
\max _{\mathcal{P}} \sum_{i=1}^{L} \mathrm{E}_{\mathbf{H}_{i}}\left\{I_{i}(\mathcal{P})\right\}
$$

subject to the same power constraints (5) and (6), where $\mathrm{E}_{\mathbf{H}_{i}}\{\cdot\}$ stands for the statistical expectation with respect to $\mathbf{H}_{i} \triangleq\left[\mathbf{H}_{i, 1}^{T}, \ldots, \mathbf{H}_{i, L}^{T}\right]^{T}$, and $(\cdot)^{T}$ denotes the matrix transpose. The above scheme (7) is useful for fast fading channels where the scheduler does not need to know any channel matrix and hence the transmitter covariance matrices are independent of the channel matrices but the receiver of each link needs to know the channel matrix of that link. The complexity of the closed form expression $\sum_{i=1}^{L} E_{\mathbf{H}_{i}}\left\{I_{i}(\mathcal{P})\right\}$ depends on the statistical properties of the channel matrices, which will be assumed to be i.i.d. Gaussian in this paper. The ergodic sum capacity is theoretically achievable through coding over a large multiple of $T$ time slots.

The implementation of the STPS scheme requires a central scheduler. In a wireless mesh network with access point (AP), the AP can take up the role of the scheduler. In an ad hoc network, the scheduler can be adaptively elected. For the case of slow fading channels, the scheduler needs to coordinate the calibration of channel matrices, collect all channel matrices, and then compute and distribute the transmitter covariance matrices of all links. In the case of fast fading channels, the scheduler does not need to known the channel matrices but assumes the statistics of all channel matrices. The statistics of all channel matrices is needed so that a closed form expression of the ergodic sum capacity $\sum_{i=1}^{L} \mathrm{E}_{\mathbf{H}_{i}}\left\{I_{i}(\mathcal{P})\right\}$ can be found to facilitate the (off line) development of the optimization algorithm.

Once each transmitter has received the information of its transmitter covariance matrices from the scheduler, the $i$ transmitter applies the transmitting covariance matrices $\mathbf{P}_{i}(t)$, $t=1, \ldots, T$ over $T$ time slots in the slow fading case or over a large multiple of $T$ time slots in the fast fading case. The $i$ th receiver decodes the packet based on $\mathbf{y}_{i}(t), t=1, \ldots, T$, and $\mathbf{H}_{i, i}$ over $T$ time slots in the slow fading case or over a large multiple of $T$ time slots in the fast fading case. With given $\mathbf{P}_{i}(t), t=1, \ldots, T$, the existing coding and modulation techniques can be applied. But the linearity of power amplifiers is required. If $T=1$ is chosen, the scheme (4) reduces to the space-only power schedule shown in [7]. If $T=1$ is chosen and $\mathbf{P}_{i}(t)$ is forced to have rank one, then both (4) and (7) become a space-only single-beam beamforming method for maximizing the sum capacity.

But (4) and (7) are appropriate only for symmetric networks where no link either dominates or is inferior to others in terms of channel quality. Otherwise, the data rate distribution among the $L$ links would be very uneven or very unfair. In the next section, we will examine a PF based STPS scheme.

There are also situations where the desired data rate for each link within a given time/frequency band is predetermined from a higher layer of the network. In such situations, we can find the transmitting covariance matrices with the least amount of total transmission power to satisfy the desired data rates for all links. The desired data rates are an important measure of QoS. In Section IV, we will present a QoS based STPS scheme.

\section{Proportional FAIR BASED STPS SCHEME}

Following the same idea as used in [12]-[14], we can revise the problem (4)-(6) into the following so called proportional fair (PF) problem for slow fading channels:

$$
\begin{array}{ll}
\max _{\mathcal{P}} & \sum_{i=1}^{L} \ln \left(I_{i}(\mathcal{P})\right) \\
\text { s.t. } & \frac{1}{T} \sum_{i=1}^{L} \sum_{t=1}^{T} \operatorname{tr}\left\{\mathbf{P}_{i}(t)\right\}=P, \quad i=1, \ldots, L
\end{array}
$$




$$
\mathbf{P}_{i}(t) \geq 0, \quad i=1, \ldots, L, \quad t=1, \ldots, T .
$$

The logarithmic function in (8) decreases the reward (i.e., the increase of data rate) for a link as the data rate of the link becomes large. In fact, the notion of "proportional fair" comes from the fact that the differential of the logarithm of a data rate is inversely proportional to the data rate. The power constraint (9) is a total power constraint which differs from the link-wise power constraint (5).

Similarly, for fast fading channels, the optimization problem with the objective function (7) and constraints (5) and (6) can be revised into:

$$
\max _{\mathcal{P}} \sum_{i=1}^{L} \ln \left(\mathrm{E}_{\mathbf{H}_{i}}\left\{I_{i}(\mathcal{P})\right\}\right)
$$

subject to constraints (9) and (10).

Both of the above optimization problems can be solved in the same way as in [9] and [10]. The logarithm in the objective function only affects the gradient computation in an obvious way. But in the following, we introduce a much simpler expression of $\mathrm{E}_{\mathbf{H}_{i}}\left\{I_{i}(\mathcal{P})\right\}$ than that used in [10], which in turn simplifies the computation of the problem (11). This simplification is based on an asymptotical form of $\mathrm{E}_{\mathbf{H}_{i}}\left\{I_{i}(\mathcal{P})\right\}$ as $N \rightarrow \infty$.

Let us write

$$
\mathbf{P}_{i}(t)=\mathbf{U}_{i}(t) \mathbf{D}_{i}(t) \mathbf{U}_{i}^{H}(t)
$$

as the eigenvalue decomposition of $\mathbf{P}_{i}(t)$, where $\mathbf{D}_{i}(t) \triangleq$ $\operatorname{diag}\left\{d_{i, 1}(t), \ldots, d_{i, N}(t)\right\}$ is the diagonal matrix of the eigenvalues and $\mathbf{U}_{i}(t)$ is the (unitary) matrix of the eigenvectors. Using (12) in (2), we have

$$
\begin{aligned}
\mathrm{E}_{\mathbf{H}_{i}}\left\{I_{i}(\mathcal{P})\right\} & \\
= & \mathrm{E}_{\mathbf{H}_{i}}\left\{\frac { 1 } { T } \sum _ { t = 1 } ^ { T } \left(\log _{2} \mid \mathbf{I}_{N}\right.\right. \\
& +\sum_{j=1}^{L} \beta_{i, j} \mathbf{H}_{i, j} \mathbf{U}_{j}(t) \mathbf{D}_{j}(t) \mathbf{U}_{j}^{H}(t) \mathbf{H}_{i, j}^{H} \mid \\
& -\log _{2} \mid \mathbf{I}_{N}+\sum_{j=1, j \neq i}^{L} \beta_{i, j} \mathbf{H}_{i, j} \mathbf{U}_{j}(t) \\
& \left.\left.\times \mathbf{D}_{j}(t) \mathbf{U}_{j}^{H}(t) \mathbf{H}_{i, j}^{H} \mid\right)\right\} .
\end{aligned}
$$

Assuming that all elements in $\mathbf{H}_{i, j}$ are i.i.d. Gaussian with zero mean and unit variance, the elements in $\tilde{\mathbf{H}}_{i, j}(t) \triangleq \mathbf{H}_{i, j} \mathbf{U}_{j}(t)$ are also i.i.d. Gaussian with zero mean and unit variance. Hence, (13) is equivalent to

$$
\begin{aligned}
\mathrm{E}_{\mathbf{H}_{i}}\left\{I_{i}(\mathcal{P})\right\} & =\mathrm{E}_{\mathbf{H}_{i}}\left\{\frac { 1 } { T } \sum _ { t = 1 } ^ { T } \left(\log _{2}\left|\mathbf{I}_{N}+\sum_{j=1}^{L} \beta_{i, j} \mathbf{H}_{i, j} \mathbf{D}_{j}(t) \mathbf{H}_{i, j}^{H}\right|\right.\right. \\
& \left.\left.-\log _{2}\left|\mathbf{I}_{N}+\sum_{j=1, j \neq i}^{L} \beta_{i, j} \mathbf{H}_{i, j} \mathbf{D}_{j}(t) \mathbf{H}_{i, j}^{H}\right|\right)\right\} .
\end{aligned}
$$

Applying [15, Theorem IV.1], which is also used in [20], (14) has the following asymptotical form with respect to $N$ :

$$
\begin{aligned}
\mathrm{E}_{\mathbf{H}_{i}}\left\{I_{i}(\mathcal{D})\right\} & \\
= & \frac{1}{T} \sum_{t=1}^{T}\left[\sum_{j=1}^{L} \sum_{n=1}^{N} \log _{2}\left(1+N \eta_{i, 1}(t) \beta_{i, j} d_{j, n}(t)\right)\right. \\
& -\sum_{j=1, j \neq i}^{L} \sum_{n=1}^{N} \log _{2}\left(1+N \eta_{i, 2}(t) \beta_{i, j} d_{j, n}(t)\right) \\
& \left.+N \log _{2} \frac{\eta_{i, 2}(t)}{\eta_{i, 1}(t)}+N\left(\eta_{i, 1}(t)-\eta_{i, 2}(t)\right) \log _{2}(e)\right]
\end{aligned}
$$

where $\mathcal{D} \triangleq\left\{d_{i, n}(t), i=1, \ldots, L, n=1, \ldots, N, t=\right.$ $1, \ldots, T\}$, and $\eta_{i, 1}(t)$ and $\eta_{i, 2}(t)$ satisfy the following nonlinear equations, respectively:

$$
\begin{aligned}
& \eta_{i, 1}(t)+\sum_{j=1}^{L} \sum_{n=1}^{N} \frac{\eta_{i, 1}(t) \beta_{i, j} d_{j, n}(t)}{N \eta_{i, 1}(t) \beta_{i, j} d_{j, n}(t)+1}=1 \\
& \eta_{i, 2}(t)+\sum_{j=1, j \neq i}^{L} \sum_{n=1}^{N} \frac{\eta_{i, 2}(t) \beta_{i, j} d_{j, n}(t)}{N \eta_{i, 2}(t) \beta_{i, j} d_{j, n}(t)+1}=1
\end{aligned}
$$

with $0<\eta_{i, 1}(t), \eta_{i, 2}(t)<1$. Since the left hand sides of (16) and (17) are monotonically increasing functions of $\eta_{i, 1}(t)$ and $\eta_{i, 2}(t)$ respectively, $\eta_{i, 1}(t)$ and $\eta_{i, 2}(t)$ can be easily found, for example, by the bisection method ([21, pp. 145]). It is worth noting that (15) is an accurate approximation to the ergodic capacity (13) even when $N$ is as small as three [20].

With the closed form expression (15) at hand, the problem (11) can be solved by the gradient projection method similar to that developed in [10], which we will not repeat.

In Section V, we will illustrate the performance of the PF-STPS scheme (8) and (11) numerically.

\section{QUALITY-OF-SERVICE BASED STPS SCHEME}

To ensure a pre-determined data rate for each link, we can formulate the QoS-STPS scheme as follows.

For slow fading channels, the QoS-STPS scheme is

$$
\begin{array}{ll}
\min _{\mathcal{P}} & \frac{1}{T} \sum_{t=1}^{T} \sum_{i=1}^{L} \operatorname{tr}\left\{\mathbf{P}_{i}(t)\right\} \\
\text { s.t. } & I_{i}(\mathcal{P}) \geq R_{i}, \quad i=1, \ldots, L \\
& \mathbf{P}_{i}(t) \geq 0, \quad i=1, \ldots, L, \quad t=1, \ldots, T
\end{array}
$$

where $R_{i}$ is the desired rate of link $i$ in bits/second/Hertz (bits/s/ $\mathrm{Hz})$. The rate $R_{i}$ can be determined by a higher layer of the network operation.

Here, we use $I_{i}(\mathcal{P})$ to measure the data rate of link $i$ as a function of the transmitter covariance matrices of all links during each time window of $T$ time slots. In practice, one can modify this function according to the actual coding and modulation methods deployed. It should also be noted that for each time window of $T$ time slots, a set of active links must be decided from a higher layer operation. Each set of active links may or 
may not overlap with other sets of active links. The construction of the sets of active links is also an important process for QoS purpose. For example, if the data rates (in bits/s/Hz averaged over $T$ time slots) of all links are the same and link $A$ requires more data to be transferred than all other links, then link $A$ should be included in more sets of active links than all other links.

Unlike that in [2] and [3] where QoS is also utilized, $\mathbf{P}_{i}(t)$ here is not constrained to have rank one. Furthermore, $\mathbf{P}_{i}(t)$ here is allowed to vary with the time slot index $t=1, \ldots, T$. With $T \geq L$, the feasible region of $R_{i}, i=1, \ldots, L$, is unbounded unless the power is bounded. A proof of this is simple. Under $T \geq L$, a sub-optimal scheduling is such that only one link is active per time slot, which is the well known TDMA. Since there is no interference in each time slot, any finite data rate is therefore feasible. But for any space-only power scheduling such as [2] and [3] where $T=1$, the feasible region of $R_{i}, i=1, \ldots, L$, is bounded (with $L>1$ ) even if the power is unbounded.

For fast fading channels, the QoS-STPS scheme follows by replacing (19) by the following:

$$
\mathrm{E}_{\mathbf{H}_{i}}\left\{I_{i}(\mathcal{P})\right\} \geq R_{i}, \quad i=1, \ldots, L .
$$

The property of the unbounded feasible region for the QoSSTPS scheme makes the optimization much easier. Since we know that the equalities in (19) and (21) are always achievable under $T \geq L$, we can follow the penalty method ([19, Sec. 3.1]) to solve the above problems as shown next.

\section{A. Slow Fading Channels}

For slow fading channels, we define the following penalized cost function:

$$
\mathcal{L}_{s}\left(\mathcal{P}, \lambda_{s}\right)=\frac{1}{T} \sum_{t=1}^{T} \sum_{i=1}^{L} \operatorname{tr}\left\{\mathbf{P}_{i}(t)\right\}+\lambda_{s} \sum_{i=1}^{L}\left(R_{i}-I_{i}(\mathcal{P})\right)^{2}
$$

where $\lambda_{s}>0$ is the associated cost coefficient. In particular, $\lambda_{s}$ starts with a small value and then gradually increases as $\mathbf{P}_{i}(t)$ is updated. The update of $\lambda_{s}$ follows a gradient ascend. The update of $\mathbf{P}_{i}(t)$ follows a projected gradient descend. With straightforward manipulations, one can verify the following recursions to minimize $\mathcal{L}_{s}\left(\mathcal{P}, \lambda_{s}\right)$ :

$$
\begin{aligned}
\lambda_{s}^{(k+1)} & =\lambda_{s}^{(k)}+\alpha_{k} \sum_{i=1}^{L}\left(Q_{i}^{(k)}\right)^{2} \\
\mathbf{P}_{i}^{(k+1)}(t) & =\left(1-\delta_{k}\right) \mathbf{P}_{i}^{(k)}(t)+\delta_{k}\left[\mathbf{P}_{i}^{(k)}(t)-s_{k} \mathbf{F}_{i}^{(k)}(t)\right]^{+}
\end{aligned}
$$

where $\alpha_{k}>0, \delta_{k}>0, s_{k}>0$ are the step-size parameters, $[\cdot]^{+}$denotes the operation of projecting an arbitrary Hermitian matrix to the set of positive semidefinite matrices, and $\mathbf{F}_{i}^{(k)}(t)$ and $Q_{i}^{(k)}$ are shown at the bottom of the page.

The parameter $\alpha_{k}$ should be chosen such that it increases with the number of iterations, while $\delta_{k}$ and $s_{k}$ can be chosen, for example, according to the Armijo rule along the feasible direction ([19, pp. 225-226]). In this rule, $s_{k}=s$ is a constant throughout the iterations, and $\delta_{k}=\theta^{m_{k}}$, where $m_{k}$ is the minimal nonnegative integer that satisfies the following inequality

$$
\begin{aligned}
\mathcal{L}_{s}\left(\mathcal{P}^{(k+1)}, \lambda_{s}^{(k)}\right)-\mathcal{L}_{s}\left(\mathcal{P}^{(k)}, \lambda_{s}^{(k)}\right) \\
\leq \sigma \theta^{m_{k}} \sum_{i=1}^{L} \sum_{t=1}^{T} \operatorname{tr}\left\{\left(\mathbf{F}_{i}^{(k)}(t)\right)^{H}\right. \\
\left.\quad \times\left(\left[\mathbf{P}_{i}^{(k)}(t)-s \mathbf{F}_{i}^{(k)}(t)\right]^{+}-\mathbf{P}_{i}^{(k)}(t)\right)\right\} .
\end{aligned}
$$

Here $\sigma$ and $\theta$ are constants. According to [19, pp. 225-226], usually $\sigma$ is chosen close to 0 , while a proper choice of $\theta$ is from 0.1 to 0.5 .

In the numerical simulations, we observe that with properly chosen step-size parameters, the sequence $\mathbf{P}_{i}^{(k)}(t)$ with respect to $k$ always converges to a local optimal solution that achieves the equality in (19). The convergence criterion is given by

$$
\max _{i \in\{1, \ldots, L\}}\left|Q_{i}^{(k)}\right| \leq \varepsilon
$$

where $\varepsilon$ is a positive constant close to zero.

In our simulations shown later, we use $s=0.1, \sigma=0.1, \theta=$ $0.5, \alpha_{k}=k$, and $\varepsilon=0.01$.

$$
\begin{aligned}
\mathbf{F}_{i}^{(k)}(t)= & \frac{\partial \mathcal{L}_{s}\left(\mathcal{P}, \lambda_{s}\right)}{\partial \mathbf{P}_{i}^{(k)}(t)} \\
= & \frac{1}{T} \mathbf{I}_{N}-\frac{2 \lambda_{s}^{(k)}}{T \ln 2}\left(Q_{i}^{(k)} \mathbf{H}_{i, i}^{H}\left[\mathbf{R}_{i}^{(k)}(t)+\beta_{i, i} \mathbf{H}_{i, i} \mathbf{P}_{i}^{(k)}(t) \mathbf{H}_{i, i}^{H}\right]^{-1} \mathbf{H}_{i, i}\right. \\
& \left.-\sum_{j=1, j \neq i}^{L} Q_{j}^{(k)} \mathbf{H}_{j, i}^{H}\left[\left(\mathbf{R}_{j}^{(k)}(t)+\beta_{j, j} \mathbf{H}_{j, j} \mathbf{P}_{j}^{(k)}(t) \mathbf{H}_{j, j}^{H}\right)^{-1}-\left(\mathbf{R}_{j}^{(k)}(t)\right)^{-1}\right] \mathbf{H}_{j, i}\right) \\
Q_{i}^{(k)}= & R_{i}-\frac{1}{T} \sum_{t=1}^{T} \log _{2}\left|\mathbf{I}_{N}+\beta_{i, i} \mathbf{H}_{i, i} \mathbf{P}_{i}^{(k)}(t) \mathbf{H}_{i, i}^{H}\left(\mathbf{R}_{i}^{(k)}(t)\right)^{-1}\right| .
\end{aligned}
$$




\section{B. Fast Fading Channels}

For fast fading channels, we will utilize the closed form expression (15). The original optimization becomes

$$
\begin{array}{ll}
\min _{\mathcal{D}} & \frac{1}{T} \sum_{t=1}^{T} \sum_{i=1}^{L} \sum_{n=1}^{N} d_{i, n}(t) \\
\text { s.t. } & \bar{I}_{i}(\mathcal{D}) \geq R_{i} \\
& d_{i, n}(t) \geq 0, i=1, \ldots, L, n=1, \ldots, N, t=1, \ldots, T
\end{array}
$$

where $\bar{I}_{i}(\mathcal{D})$ is the right side of $(15)$.

Now using the penalty method, we define the cost function $\mathcal{L}_{f}\left(\mathcal{D}, \lambda_{f}\right)$ as follows:

$$
\mathcal{L}_{f}\left(\mathcal{D}, \lambda_{f}\right)=\frac{1}{T} \sum_{t=1}^{T} \sum_{i=1}^{L} \sum_{n=1}^{N} d_{i, n}(t)+\lambda_{f} \sum_{i=1}^{L}\left(R_{i}-\bar{I}_{i}(\mathcal{D})\right)^{2}
$$

where $\lambda_{f}>0$ is the associated cost coefficient.

The stationary point of $\mathcal{L}_{f}\left(\mathcal{D}, \lambda_{f}\right)$ can be found by an iterative method, where at the $k$ th iteration, $\lambda_{f}^{(k)}$ and $d_{i, n}^{(k)}(t)$ are updated in the positive and negative gradient directions respectively as follows:

$$
\begin{aligned}
\lambda_{f}^{(k+1)}= & \lambda_{f}^{(k)}+\alpha_{k} \sum_{i=1}^{L}\left(W_{i}^{(k)}\right)^{2} \\
d_{i, n}^{(k+1)}(t)= & \left(1-\delta_{k}\right) d_{i, n}^{(k)}(t) \\
& +\delta_{k}\left[d_{i, n}^{(k)}(t)-s_{k} \frac{\partial \mathcal{L}_{f}\left(\mathcal{D}, \lambda_{f}\right)}{\partial d_{i, n}^{(k)}(t)}\right]^{+}
\end{aligned}
$$

where

$$
\begin{aligned}
& \frac{\partial \mathcal{L}_{f}\left(\mathcal{D}, \lambda_{f}\right)}{\partial d_{i, n}^{(k)}(t)} \\
& =\frac{1}{T}+\frac{2 \lambda_{f}^{(k)}}{T \ln 2}\left[\sum_{j=1, j \neq i}^{L} W_{j}^{(k)}\right. \\
& \quad \times\left(\frac{N \eta_{j, 2}^{(k)}(t) \beta_{j, i}}{1+N \eta_{j, 2}^{(k)}(t) \beta_{j, i} d_{i, n}^{(k)}(t)}\right. \\
& \left.\left.\quad-\frac{N \eta_{j, 1}^{(k)}(t) \beta_{j, i}}{1+N \eta_{j, 1}^{(k)}(t) \beta_{j, i} d_{i, n}^{(k)}(t)}\right)\right] \\
& \quad-\frac{2 \lambda_{f}^{(k)}}{T \ln 2} W_{i}^{(k)}\left(\frac{N \eta_{i, 1}^{(k)}(t) \beta_{i, i}}{1+N \eta_{i, 1}^{(k)}(t) \beta_{i, i} d_{i, n}^{(k)}(t)}\right) \\
& W_{i}^{(k)}=R_{i}-\bar{I}_{i}\left(\mathcal{D}^{(k)}\right) .
\end{aligned}
$$

Here the step-size parameters can be chosen similarly as in Section IV-A. In the numerical simulations, we observe that with properly chosen step-size parameters, the sequence $d_{i, n}^{(k)}(t)$ always converges to a local optimal solution which satisfies the equality in (23).

\section{NUMERICAL EXAMPLES}

We now illustrate the performance of the PF-STPS scheme shown in Section III and the QoS-STPS scheme developed in Section IV. Throughout the simulations, we set $T=L$.

\section{A. Performance of the PF-STPS Scheme}

For convenience of comparison, we will refer to the sum capacity based STPS scheme shown in Section II as SC-STPS(A). We will also consider the sum capacity based STPS with total power constraint (9), instead of link-wise power constraint (5), which will be referred to as SC-STPS(B).

For both slow and fast fading channels, we define the signal-to-noise ratio (SNR) of the $i$ th link as $\mathrm{SNR}_{i} \triangleq$ $\beta_{i, i} P /(N L)$ and the nominal interference-to-noise ratio (INR) from the $j$ th transmitting node to the $i$ th receiving node as $\mathrm{INR}_{i, j} \triangleq \beta_{i, j} P /(N L)$. We set $P_{i}=P / L, i=1, \ldots, L$. We consider an asymmetric network with $L / 2$ strong links and $L / 2$ weak links, i.e.,

$$
\mathrm{SNR}_{i}= \begin{cases}30 \mathrm{~dB} & i=1, \ldots, \frac{L}{2} \\ 20 \mathrm{~dB} & i=\frac{L}{2}+1, \ldots, L\end{cases}
$$

We also set $\operatorname{INR}_{i, j}=\mathrm{INR}, i, j=1, \ldots, L, i \neq j$ and vary INR in each example.

First, we simulate the slow fading channels where all channel matrices $\mathbf{H}_{i, j}, i, j=1, \ldots, L$ are known at the scheduler. We also assume that all elements in the channel matrices are quasi-static, which remain constant within each block of $T$ time slots but change independently from block to block with i.i.d. Gaussian distribution.

The performance of all algorithms are compared in terms of (a) the per link capacity averaged over strong links, (b) the per link capacity averaged over weak links, and (c) the per link capacity averaged over all links, defined as follows:

$$
\begin{gathered}
C_{\text {strong }}^{\text {slow }} \triangleq \frac{2}{J L} \sum_{j=1}^{J} \sum_{i=1}^{\frac{L}{2}} I_{i}^{(j)}\left(\mathcal{P}^{*}\right) \\
C_{\text {weak }}^{\text {slow }} \triangleq \frac{2}{J L} \sum_{j=1}^{J} \sum_{i=\frac{L}{2}+1}^{L} I_{i}^{(j)}\left(\mathcal{P}^{*}\right) \\
C_{\text {all }}^{\text {slow }} \triangleq \frac{1}{J L} \sum_{j=1}^{J} \sum_{i=1}^{L} I_{i}^{(j)}\left(\mathcal{P}^{*}\right)
\end{gathered}
$$

where $I_{i}^{(j)}\left(\mathcal{P}^{*}\right)$ denotes the $i$ th link capacity (2) with the converged $\mathcal{P}$ for the $j$ th channel realization. We use $J=100$ channel realizations. Obviously, we have $C_{\text {all }}^{\text {slow }}=\left(C_{\text {strong }}^{\text {slow }}+\right.$ $\left.C_{\text {weak }}^{\text {slow }}\right) / 2$.

Fig. 2 illustrates the per link (mean) capacity of SC-STPS(A), SC-STPS(B) and PF-STPS with $N=4$ and $L=T=10$. In terms of $C_{\text {all }}^{\text {slow }}$, the three schemes are about the same although SC-STPS(B) is slightly better than others. Both SC-STPS(A) and SC-STPS(B) maximize the sum capacity. But SC-STPS(B) is subject to a weaker power constraint. Hence, SC-STPS(B) is expected to yield a higher $C_{\text {all }}^{\text {slow }}$ than SC-STPS(A). PF-STPS 


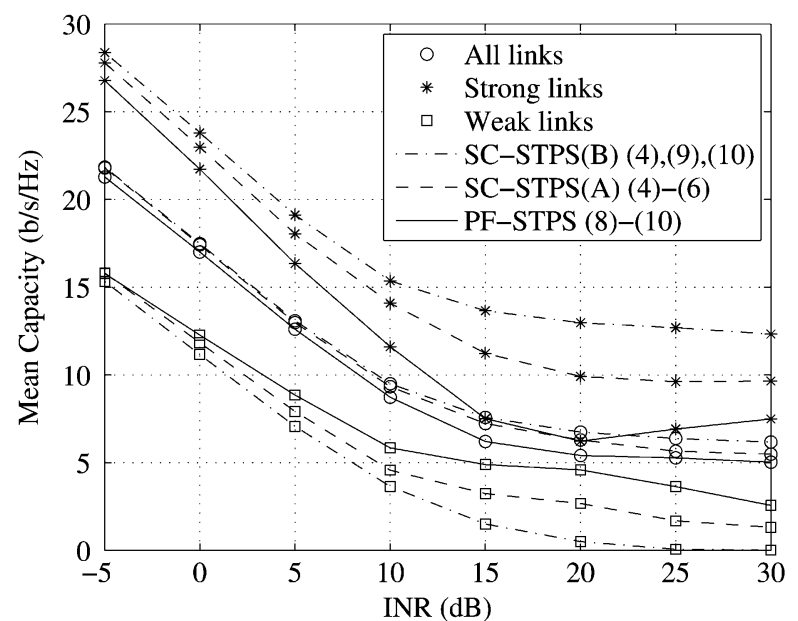

Fig. 2. Per link capacity comparison of the SC-STPS(A), SC-STPS(B), and PF-STPS algorithms. Slow fading. $N=4, L=T=10$.

is not designed to maximize the sum capacity and hence is expected to yield lower $C_{\text {all }}^{\text {slow }}$ than the other two schemes. However, this numerical example shows that the differences in $C_{\text {all }}^{\text {slow }}$ among the three schemes are very small. This phenomenon is also confirmed by other examples in our simulations. Fig. 2 also shows that the difference $C_{\mathrm{strong}}^{\text {slow }}-C_{\text {weak }}^{\text {slow }}$ is the smallest for PF-STPS, the second for SC-STPS(A) and the largest for SC-STPS(B). This is expected from the problem formulation of each scheme. But this numerical example shows that $C_{\mathrm{strong}}^{\text {slow }}-$ $C_{\text {weak }}^{\text {slow }}$ varies significantly among the three schemes. Other examples in our simulation also support this observation. Therefore, PF-STPS appears to have the best tradeoff between sum capacity and fairness among the three schemes.

Next, we consider the fast fading channels. In this case, we define the following performance measures:

$$
\begin{aligned}
C_{\text {strong }}^{\text {fast }} \triangleq \frac{2}{L} \sum_{i=1}^{\frac{L}{2}} \mathrm{E}_{\mathbf{H}_{i}}\left\{I_{i}\left(\mathcal{P}^{*}\right)\right\} \\
C_{\text {weak }}^{\text {fast }} \triangleq \frac{2}{L} \sum_{i=\frac{L}{2}+1}^{L} \mathrm{E}_{\mathbf{H}_{i}}\left\{I_{i}\left(\mathcal{P}^{*}\right)\right\} \\
C_{\text {all }}^{\text {fast }} \triangleq \frac{1}{L} \sum_{i=1}^{L} \mathrm{E}_{\mathbf{H}_{i}}\left\{I_{i}\left(\mathcal{P}^{*}\right)\right\}
\end{aligned}
$$

where $\mathcal{P}^{*}$ denotes the converged $\mathcal{P}$ by the fast fading algorithms, and $\mathrm{E}_{\mathbf{H}_{i}}$ here is performed by averaging over 1000 independent realizations of i.i.d. Gaussian channel matrices with each given $\mathcal{P}^{*}$.

Fig. 3 illustrates the per link (mean) capacity of SC-STPS(A), SC-STPS(B) and PF-STPS with $N=2$ and $L=T=6$ in the fast fading case. The general phenomenon observed here is the same as in the slow fading case.

\section{B. Performance of the QoS-STPS Scheme}

We evaluate the performance of the QoS-STPS scheme for two different network settings:

- Symmetric network: The channel gains for all desired links are $\beta_{i, i}=\rho, i=1, \ldots, L$, and the channel gains for all

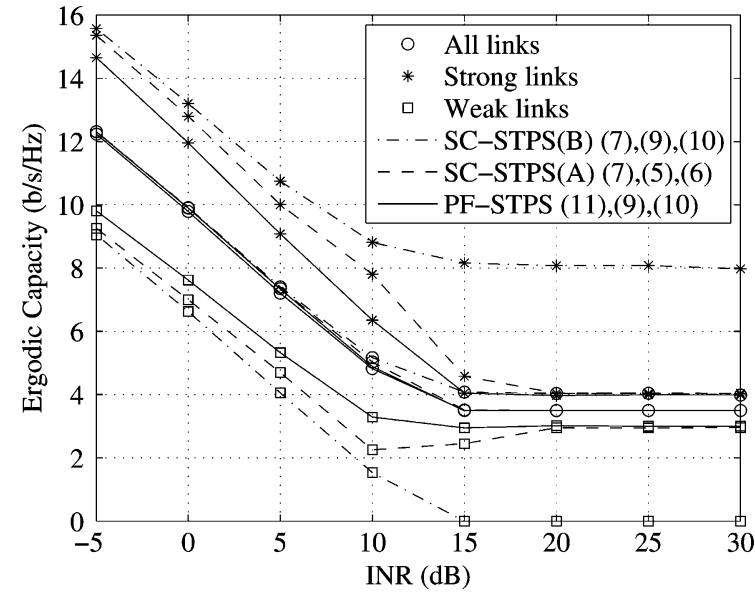

Fig. 3. Per link capacity comparison of the SC-STPS(A), SC-STPS(B), and PF-STPS algorithms. Fast fading. $N=2, L=T=6$.

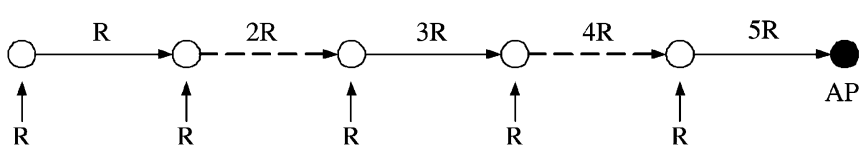

Fig. 4. An asymmetric network: each node collects information at the rate $R$ and forwards the information to the access point (AP). The capacity of the three active links (solid-line arrows) within a given time/frequency band must equal to $R, 3 R$, and $5 R$, respectively. The other two links (dash-line arrows) must occupy a different time/frequency band so that no node transmits and receives in the same time/frequency band. These two links are not considered in the simulation.

interfering links are $\beta_{i, j}=\beta, i, j=1, \ldots, L, j \neq i$, and the rate requirement is $R_{i}=R, i=1, \ldots, L$.

- Asymmetric network: Five nodes are uniformly and linearly aligned together, forming a chain of relays accessing to a common access point (AP) as shown in Fig. 4. We consider power scheduling for the three links as indicated by the solid arrows in a given time/frequency band. (Given no node can transmit and receive in the same time/frequency band, the other two links indicated by the dash arrows would have to be scheduled in a different time/frequency band.) The rate distribution for the three links are $R_{1}=R, R_{2}=3 R$, and $R_{3}=5 R$. This is a simple example of wireless mesh network with access point.

For slow fading channels, we compare our slow fading QoS-STPS scheme (18)-(20) with the slow fading single-beam beamforming algorithm developed in [2]. For fast fading channels, we compare our fast fading QoS-STPS scheme (22)-(24) with the following fast fading single-beam beamforming alternative:

$$
\begin{aligned}
\min _{\mathbf{p}} & \sum_{i=1}^{L} p_{i} \\
\text { s.t. } & \mathrm{E}_{\mathbf{h}_{i}}\left\{\log _{2}\left(1+\beta_{i, i} p_{i} \mathbf{h}_{i, i}^{H} \mathbf{S}_{i}^{-1} \mathbf{h}_{i, i}\right)\right\} \geq R_{i}, \\
& p_{i} \geq 0, \quad i=1, \ldots, L
\end{aligned}
$$




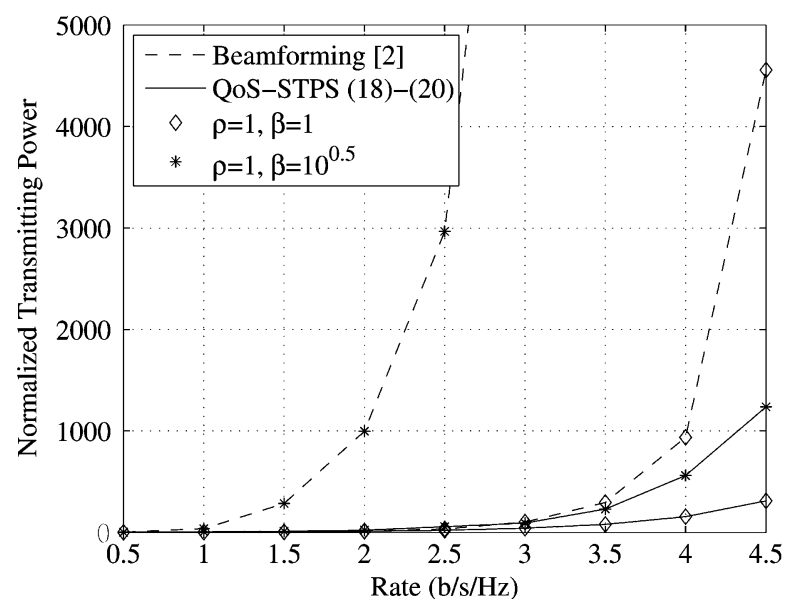

Fig. 5. Sum power comparison between the QoS-beamforming and the QoSSTPS algorithms. Symmetric network. Slow fading. $N=4$ and $L=T=10$.

where $\mathbf{S}_{i}=\sum_{j=1, j \neq i}^{L} p_{j} \beta_{i, j} \mathbf{h}_{i, j} \mathbf{h}_{i, j}^{H}+\mathbf{I}_{N}$ is the interferenceplus-noise covariance matrix at the $i$ th receiving node when the single-beam beamforming approach is applied at all transmitting nodes, $\mathbf{h}_{i, j}$ is an $N \times 1$ equivalent channel vector, $\mathbf{h}_{i} \triangleq$ $\left[\mathbf{h}_{i, 1}^{T}, \ldots, \mathbf{h}_{i, L}^{T}\right]^{T}$, and $\mathbf{p} \triangleq\left[p_{1}, \ldots, p_{L}\right]^{T}$ is a vector containing all power allocation parameters. Note that for fast fading channels, we assume that the transmitter covariance matrices are independent of the channel matrices. For any given rank-one transmitter covariance matrix, there is one transmitting beam vector. The equivalent channel vector of a link is simply the channel matrix of the link multiplied by the transmitting beam vector of the same link.

For the symmetric network and slow fading channels, Fig. 5 shows the total power (averaged over 100 channel realizations) required by the (slow fading) QoS-beamforming scheme in [2] and our (slow fading) QoS-STPS scheme versus the data rate $R$, where $N=4, T=L=10$ and $\rho=1$ while $\beta=1$ or $\beta=\sqrt{10}$. With $\beta=1$, the nominal interference level is comparable to the desired signal level $\rho=1$. With $\beta=$ $\sqrt{10}$, the nominal interference level is higher than the desired signal level $\rho=1$. In either case, the QoS-STPS scheme consumes much less power than the QoS-beamforming scheme. In the case of $\beta=\sqrt{10}$, the data rate $R=2.5 \mathrm{~b} / \mathrm{s} / \mathrm{Hz}$ is nearly infeasible for the QoS-beamforming scheme but is still well within the feasible region for the QoS-STPS scheme. This significant advantage of the QoS-STPS scheme over the QoSbeamforming scheme is due to two reasons. The first is that the QoS-STPS scheme fully utilizes the spatial freedom while the QoS-beamforming scheme only exploits a single stream per link. The second is that the QoS-STPS scheme optimally integrates link scheduling (temporal freedom) with MIMO power control (spatial freedom) while the QoS-beamforming does not exploit the temporal freedom at all.

For the asymmetric network and slow fading channels, Fig. 6 shows the total power required by QoS-beamforming and QoSSTPS versus the data rate $R$. The advantage of QoS-STPS is obvious.

For the symmetric network and fast fading channels, we select $\rho=\beta=1$ and Fig. 7 shows the sum power required by QoS-beamforming and QoS-STPS versus the data rate $R$.

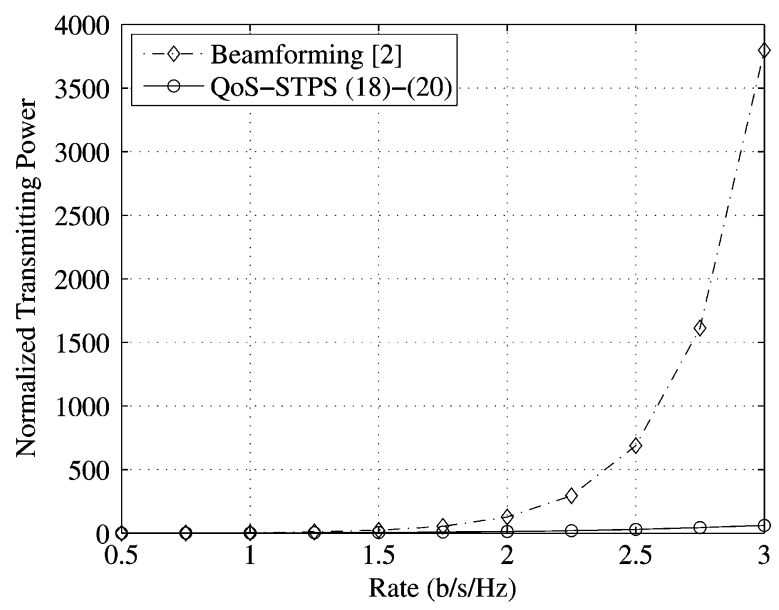

Fig. 6. Sum power comparison between the QoS-beamforming and the QoSSTPS algorithms. Asymmetric network. Slow fading. $N=4$ and $L=T=3$.

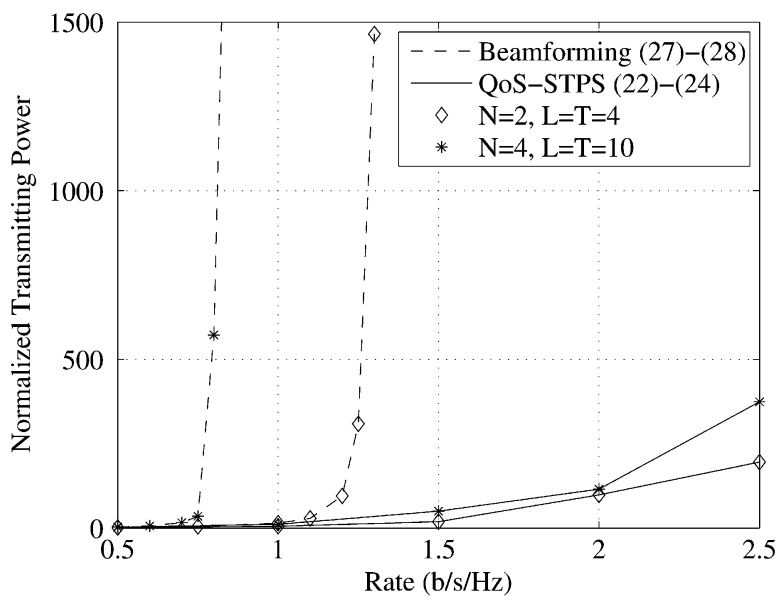

Fig. 7. Sum power comparison between the QoS-beamforming and the QoSSTPS algorithms. Symmetric network. Fast fading. $\rho=\beta=1$.

This figure highlights a fact that the performance difference between the two schemes increases dramatically as $N, L$, and $T$ increase. In particular, we see that the feasible region of data rate for QoS-beamforming shrinks quickly as $N, L$, and $T$ increase.

For the asymmetric network and fast fading channels, we select $N=4$ and $T=L=3$ and Fig. 8 shows the sum power required by QoS-beamforming and QoS-STPS versus the data rate $R$.

It is useful to observe from Figs. 5-8 that at a moderate power consumption, the QoS-STPS scheme can allow each link to operate at a relatively high spectral efficiency in bits/s/Hz. This suggests that the advanced physical layer technology of MIMO transceivers can be deployed along with the QoS-STPS scheme.

\section{CONCLUSION}

In this paper, we have investigated the fairness and quality-of-service (QoS) issues of the space-time power scheduling (STPS) scheme for a network of distributed MIMO links with a central scheduler. This scheduler can be elected adaptively among any eligible nodes in the network. To solve 


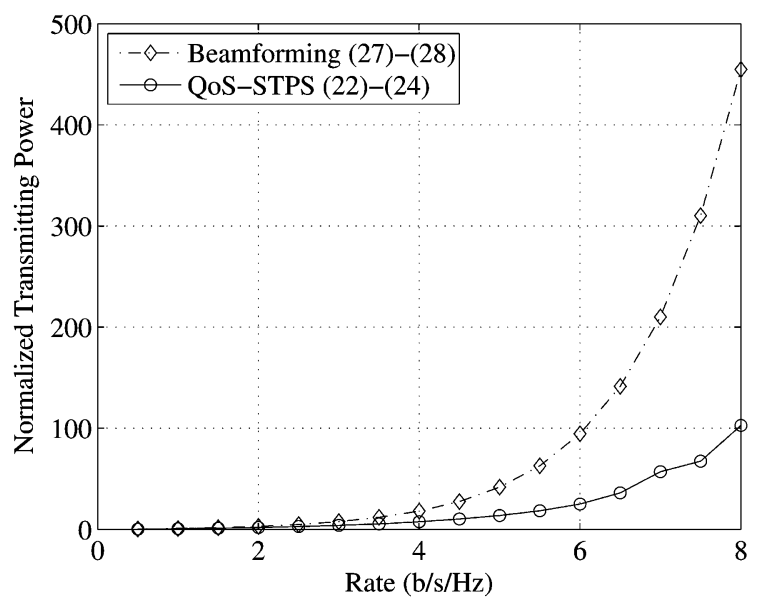

Fig. 8. Sum power comparison between the QoS-beamforming and the QoSSTPS algorithms. Asymmetric network. Fast fading. $N=4$ and $L=T=3$.

the fairness issue in the case of asymmetric networks, we have examined the proportional fair (PF) criterion. As shown through simulation, the PF-STPS scheme provides a very attractive tradeoff between sum capacity and fair distribution of data rates in comparison to two other alternatives that directly maximize the sum capacity. One innovation in this part of this paper is a simplified closed form expression of the ergodic capacity for fast fading channels, which simplifies the optimization algorithm significantly. For the QoS issue, we have developed the QoS-STPS scheme that minimizes the total transmission power subject to data rate constraints. Within the STPS framework where the transmitter covariance matrices are functions of time and/or frequency, any set of finite link data rates in any given time/frequency band can be feasible for a finite network. We have exploited this property to transform the original optimization problem into one much easier to solve. This transformation would not have been possible without the STPS framework. Simulation results consistently show that the QoS-STPS scheme outperforms the QoS-beamforming scheme in [2]. This paper has further demonstrated the importance of the STPS scheme which optimally integrates link scheduling, multiple-beam beamforming and power control.

It is anticipated that the STPS scheme can be applied to improve many existing schemes and algorithms for multi-antenna and multi-user systems. One such application can be singlebeam beamforming based STPS, which would retain part of the simplicity of single-beam beamforming and at the same time utilize the advantage of joint design of link scheduling and power control. This would be a generalization of [2]. Another application is to consider practical coding and modulation constraints for the STPS scheme.

The computational complexity of the STPS scheme needs further research as it affects the size of the network to which the STPS scheme can be applied in practice. The larger is the network scheduled by the STPS scheme within a single time/frequency band, the higher is the overall network power-and-spectral efficiency.

Future research should also include a detailed evaluation of the requirements for the STPS framework. The validity of the knowledge of all channel matrices at the scheduler for slow fading channels needs further examination. The validity of the statistical channel model used for fast fading channels also requires further attention. Research in this direction requires multi-disciplinary efforts.

\section{REFERENCES}

[1] V. Stankovic, A. Host-Madsen, and Z. Xiong, "Cooperative diversity of wireless ad hoc networks," IEEE Signal Process. Mag., vol. 23, no. 5, pp. 37-49, Sept. 2006.

[2] J.-H. Chang, L. Tassiulas, and F. Rashid-Farrokhi, "Joint transmitter receiver diversity for efficient space division multiaccess," IEEE Trans. Wireless Commun., vol. 1, pp. 16-27, Jan. 2002.

[3] A. Pascual-Iserte, A. I. Pérez-Neira, and M. Á. Lagunas, "An approach to optimum joint beamforming design in a MIMO-OFDM multiuser system," EURASIP J. Wireless Commun. Networking, no. 2, pp. 210-221, 2004.

[4] B. Song, R. L. Cruz, and B. D. Rao, "Network duality for multiuser MIMO beamforming networks and applications," IEEE Trans. Commun., vol. 55, pp. 618-630, Mar. 2007.

[5] M. F. Demirkol and M. A. Ingram, "Power-controlled capacity for interfering MIMO links," in Proc. IEEE VTC, Atlantic City, NJ, Oct. 2001, vol. 1, pp. 187-191.

[6] R. S. Blum, "MIMO capacity with interference," IEEE J. Sel. Areas Comm., vol. 21, pp. 793-801, June 2003.

[7] S. Ye and R. S. Blum, "Optimized signaling for MIMO interference systems with feedback," IEEE Trans. Signal Process., vol. 51, pp. 2839-2848, Nov. 2003.

[8] O. Popescu, D. C. Popescu, and C. Rose, "Simultaneous water filling in mutually interfering systems," IEEE Trans. Wireless Commun., vol. 6, pp. 1102-1113, Mar. 2007.

[9] Y. Rong and Y. Hua, "Optimal power schedule for distributed MIMO links," IEEE Trans. Wireless Commun., to be published, in Proc. 25th Army Science Conf. (short version), Orlando, FL, Nov. 27-30, 2006.

[10] Y. Rong, Y. Hua, A. Swami, and A. L. Swindlehurst, "Space-time power schedule for distributed MIMO links without instantaneous channel state information at the transmitting nodes," IEEE Trans. Signal Process., vol. 56, pp. 686-701, Feb. 2008.

[11] F. Kelly, "Charging and rate control for elastic traffic," Eur. Trans. Telecommun., vol. 8, pp. 33-37, Jan. 1997.

[12] A. Jalali, R. Padovani, and R. Pankaj, "Data throughput of CDMA-HDR a high efficiency-high data rate personal communication wireless system," in Proc. IEEE Veh. Technol. Conf., 2000, pp. 1854-1858

[13] K.-D. Lee and V. C. M. Leung, "Fair allocation of subcarrier and power in an OFDMA wireless mesh network," IEEE J. Select. Areas Commun., vol. 24, no. 11, pp. 2051-2060, Nov. 2006.

[14] V. K. N. Lau, "Proportional fair space-time scheduling for wireless communications," IEEE Trans. Commun., vol. 53, pp. 1353-1360, Aug. 2005.

[15] D. N. C. Tse and S. V. Hanly, "Linear multiuser receivers: Effective interference, effective bandwidth and user capacity," IEEE Trans. Inform. Theory, vol. 45, no. 2, pp. 641-657, Mar. 1999.

[16] G.-S. Kuo, A. Mishra, and R. Prasad, "QoS and resource allocation in 3rd-generation wireless networks [Guest Editorial]," IEEE Commun. Mag., vol. 39, p. 115, Feb. 2001.

[17] U. C. Kozat, I. Koutsopoulos, and L. Tassiulas, "Cross-layer design for power efficiency and QoS provisioning in multi-hop wireless networks," IEEE Trans. Wireless Commun., vol. 5, pp. 3306-3315, Nov. 2006.

[18] M. Johansson and M. Sternad, "Resource allocation under uncertainty using the maximum entropy principle," IEEE Trans. Inform. Theory, vol. 51, pp. 4103-4117, Dec. 2005.

[19] D. P. Bertsekas, Nonlinear Programming, 2nd ed. Belmont, MA: Athena Scientific, 1995.

[20] A. Lozano and A. M. Tulino, "Capacity of multiple-transmit multiplereceive antenna architectures," IEEE Trans. Inform. Theory, vol. 48, pp. 3117-3128, Dec. 2002.

[21] S. Boyd and L. Vandenberghe, Convex Optimization. Cambridge, U.K.: Cambridge Univ. Press, 2004. 


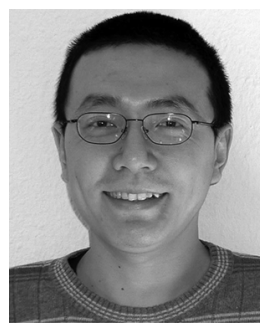

Yue Rong (S'03-M'06) was born in Jiangsu, China, in 1976. He received the B.E. degree from Shanghai Jiao Tong University, China, the M.Sc. degree from the University of Duisburg-Essen, Duisburg, Germany, and the Ph.D. degree (summa cum laude) from Darmstadt University of Technology, Darmstadt, Germany, all in electrical engineering, in 1999, 2002, and 2005, respectively.

From April 2001 to October 2001, he was a Research Assistant at the Fraunhofer Institute of Microelectronic Circuits and Systems, Duisburg, Germany. From October 2001 to March 2002, he was with Nokia Ltd., Bochum, Germany. From November 2002 to March 2005, he was a Research Associate at the Department of Communication Systems, University of Duisburg-Essen. From April 2005 to January 2006, he was with the Institute of Telecommunications at Darmstadt University of Technology, as a Research Associate. From February 2006 to November 2007, he was a Postdoctoral Researcher with the Department of Electrical Engineering, University of California, Riverside. Since December 2007, he has been with the Department of Electrical and Computer Engineering, Curtin University of Technology, Perth, Australia, as a Lecturer. His research interests include signal processing for communications, wireless communications, wireless networks, applications of linear algebra and optimization methods, and statistical and array signal processing.

Dr. Rong received the 2001-2002 Graduate Sponsoring Asia scholarship of DAAD/ABB (Germany) and the 2004 Chinese Government Award for Outstanding Self-Financed Students Abroad (China).

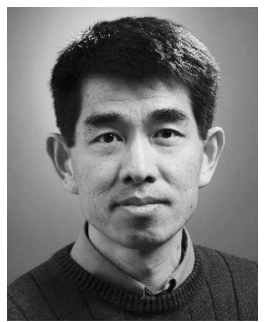

Yingbo Hua (S'86-M'88-SM'92-F'02) received B.E. degree in Feb. 1982 from Nanjing Institute of Technology (Southeast University), Nanjing, China, and M.S. degree in 1983 and Ph.D. degree in 1988 from Syracuse University, Syracuse, NY. He was supported by Chinese Government Scholarship and Syracuse University Graduate Fellowship.

In February 1990, he joined the faculty of the University of Melbourne, Melbourne, Australia, where he stayed until 2001. Since February 2001, he has been Professor of Electrical Engineering with the University of California, Riverside, CA. He has published over 260 articles in journals, conference proceedings and books in areas such as sensor array and high-resolution signal processing, principal component analysis and reduced rank filtering, blind system identification and source separations, cooperative wireless relay networks, and throughput enhancement for large-scale wireless networks. He co-edited two books.

Dr. Hua has served on Editorial Boards of IEEE TRANSACTIONS ON Signal Processing, IEEE Signal Processing LetTers, Signal Processing (EURASIP) and IEEE Signal Processing Magazine. He has also served as Guest Editor for two IEEE Signal Processing Magazine Special Issues. He was a member on IEEE Signal Processing Society's Technical Committees for Underwater Acoustic Signal Processing, Sensor Array and Multi-Channel Signal Processing, and Signal Processing for Communications and Networking. He has served on numerous technical program committees of international conferences and workshops. 